\title{
Synthetic Vision: A Prototype Display Concept for Commercial Aircraft
}

\author{
E.Theunissen, R.M.Rademaker \\ Delft University of Technology \\ $\&$ \\ T.J. Etherington \\ Collins Advanced Technology Center
}

\begin{abstract}
The synthetic vision implementation discussed in this paper comprises elements in the navigation display, the vertical profile display, and the primary flight display. The design rationale is provided based on an overview of the intended functions, options to implement these functions, and the justifications used in the selection of a specific option.
\end{abstract}

\section{INTRODUCTION}

Spatial displays for guidance and navigation are featured in several science fiction movies, the most famous of which is probably Stanley Kubrick's, 2001 - A Space Odyssey, based on the novel by Arthur C. Clarke. In 2001, astronauts use a perspective display showing the desired trajectory during a Moon landing.

Although spatial displays are not yet present in the flightdeck of today's aircraft, their potential for threat detection and avoidance, navigation, guidance, and control was already recognized more than forty years ago and has been addressed in many studies [1-4]. In 2001, - a concept of operations for commercial and business aircraft synthetic vision systems was published [5].

The absence of perspective flight path displays in commercial aircraft may soon be something of the past. In May 1999, a team headed by Rockwell Collins, Inc., was awarded a contract for the development and implementation of a synthetic vision system for business and commercial aviation. Based on results from earlier studies performed by Rockwell Collins and Delft University, a prototype of the head-down display concept for a synthetic vision information system (SVIS) has been

Author's Current Address:

E. Theunissen and R.M. Rademaker, Delft University of Technology, Information Technology and Systems, Department of Electrical Engineering, PO Box 5031, $2600 \mathrm{GA}$ Delft, The Netherlands; T.J. Etherington, Collins Advanced Technology Center, Cedar Rapids, IA, USA.

Based on a presentation at DASC 2001 .

$0885 / 8985 / 02 / \$ 17.00 @ 2002$ IEEE jointly developed. It is an evolutionary concept which combines an artificial presentation of the terrain for awareness purposes with a depiction of the planned trajectory and augmentation symbology to support the pilot with the guidance and control task.

Previous research has addressed the potential of the various elements considered part of a synthetic vision system with respect to safety and operational capabilities [1-4]. This paper outlines the design philosophy of the synthetic vision display formats developed by the Collins team. After a brief summary of important results from earlier research that form the basis of the current concept, this paper discusses the intended functions of SVIS and the elements used to realize these functions.

\section{EARLIER RESEARCH}

The current paper discusses three formats of the SVIS: The egocentric guidance format on the Primary Flight Display (PFD); the plan view with integrated terrain on the Navigation Display (ND); and the profile view on the Vertical Profile Display (VPD). None of these formats is "new" in a sense that it has never been implemented before, but each contains a number of specific features that are unique. Synthetic-vision research mainly focuses on elements in the PFD $[2,4]$. To this authors' knowledge, the current implementation is the first in which all three display formats are integrated into a synthetic vision information system.

\section{Vertical Profile Display}

The concept of the VPD was already discussed and investigated in 1976 [6]. This early version depicted a side view of the planned path relative to the current position of the aircraft and included indications of both flight path and potential flight path angle. Although the format discussed did not include a depiction of the underlying terrain, it was indicated that "significant terrain features can be shown referenced to the moving tape. Logic will have to be provided to change these features as a function of lateral displacement from the desired ground track." 
Integrating terrain in this type of display format requires a decision to be made with respect to the terrain depicted, i.e., below the current track or below the planned path. When using a track-based VPD to fly a planned LNAV path, the terrain profile will continuously change in turns, and suggest conflicts which will never arise when correctly following the path. A VPD that shows an alongtrack view does not suffer from this problem, but when the aircraft strays away from the path, the depicted profile does not represent the real situation. Thus, the optimal VPD mode depends on mode of operation.

\section{Terrain Integration in Plan View Displays}

Research into the integration of terrain contour data on the navigation display demonstrated that this increases the likelihood of detection of trajectories which result in a Controlled Flight Into Terrain (CFIT). Kuchar and Hansman [7] compared three different terrain alerting display formats and report that with the plan view display, $80 \%$ of the time lateral maneuvers were executed versus $30 \%$ of the time with a perspective display and $5 \%$ of the time with a vertical profile display. From these studies, it can be concluded that an emphasis on a certain spatial dimension causes a bias in the decisions, and certainly not always yields the best solution. Therefore, the depiction of terrain should not be restricted to solely the lateral or the vertical plane.

Kuchar and Hansman [7] also investigated the effectiveness of terrain color coding. They report that color coding relative to the aircraft yielded a more intuitive depiction of terrain hazards, and color coding relative to MSL can have merit for strategic planning, e.g., in engine-out forced descents.

\section{Perspective Flight Path Displays}

Perspective flight path displays were already evaluated in simulators and flight tested in the early 1980s [1]. These studies were proof-of-concept-oriented. Comparison studies with conventional display formats showed improved tracking performance, better situation awareness, and lower workload with perspective flight path displays. It is also reported that the advantages become more pronounced with increasing complexity of the path. Each of the previous studies used a different implementation and different values for the design parameters, and no design rationale is provided for the selection of the values of these parameters. In order to base future designs on specific requirements, Theunissen [3] focused on describing the influence of the design parameters and display augmentation on control strategies, tracking performance, and control activity. Meanwhile, the proof-of-concept and the demonstration of potential advantages has been repeatedly shown by many other studies.

In summary, a well-designed pathway display can contribute both to an improvement of navigation awareness and to the ability to accurately fly complex paths with acceptable workload. The main advantages in the area for manual control relative to current command displays result from the fact that because of the integrated trajectory preview, an anticipatory control strategy can be applied and the pilot gain for closed-loop compensatory control can be lower. This is a more effective form of control as compared to pure compensatory tracking of commands.

\section{Synthetic Vision}

Although there is no single agreed-upon definition of synthetic vision, the common element in almost all definitions and implementations is a computer-generated depiction of the environment based on spatial data. Almost all of the synthetic vision systems proposed aim to provide an artificial presentation of the outside world as seen from the cockpit $[2,4,5]$.

Gordon [8] points out some basic questions that need to be answered before a synthetic vision system is developed. The first: Is the synthetic vision display meant to be an improvement on current primary flight displays (PFDs), showing attitude (including terrain), airspeed, altitude, vertical speed, heading and navigation information? addresses both the definition of synthetic vision and the type of operation being envisioned. Several SVS concepts discuss operations in which the synthetic image is meant as a replacement of the out-of-the-windshield view $[2,4]$.

With respect to the integration of terrain information in the PFD, Gordon [8] continues: If so, is the introduction of realistic-looking graphics representing terrain surface features (oceans, mountains, rivers, etc.), and geo-political boundaries on a PFD a good idea?'

Posed like this, it is almost rhetorical, and perhaps rightly so. This issue was also addressed by Theunissen [9], who argues that "the level of realism conveyed by certain presentations can increase confidence in the conveyed information to a level which cannot be justified from the integrity of the data. Such unjustified confidence can result in a confirmation bias. Unintended use, combined with a confirmation bias and an error in the data can result in CFIT."

A relatively abstract representation of the terrain should be sufficient to provide terrain awareness. Recent research results suggest that when the task requires recognition of the geometric structure of the environment but not the information contained in the phototexture, the use of a well-designed regular texture pattern is more beneficial.

For systems meant to replace the pilot's current out-of-the-windshield view, Gordon asks: To what degree of fidelity should the outside world be portrayed to be capable of replacing the current pilot's eye view? That the situation is even more challenging is indicated by his remark that "Reducing the entire outside world to the size of a single piece of glass in the cockpit is the type of aggressive goal that begs for initial human factors review."

In contrast, the system discussed in this paper is not intended to be a sole-means system, in a sense that it can be used to replace the outside world as viewed through the windscreen. It complements and enhances the information conveyed by the view of the outside world; however, this is only one element. In today's operations, even with perfect visibility, the out-of-the-windshield view is one of many information sources used for navigation. It allows the pilot to 


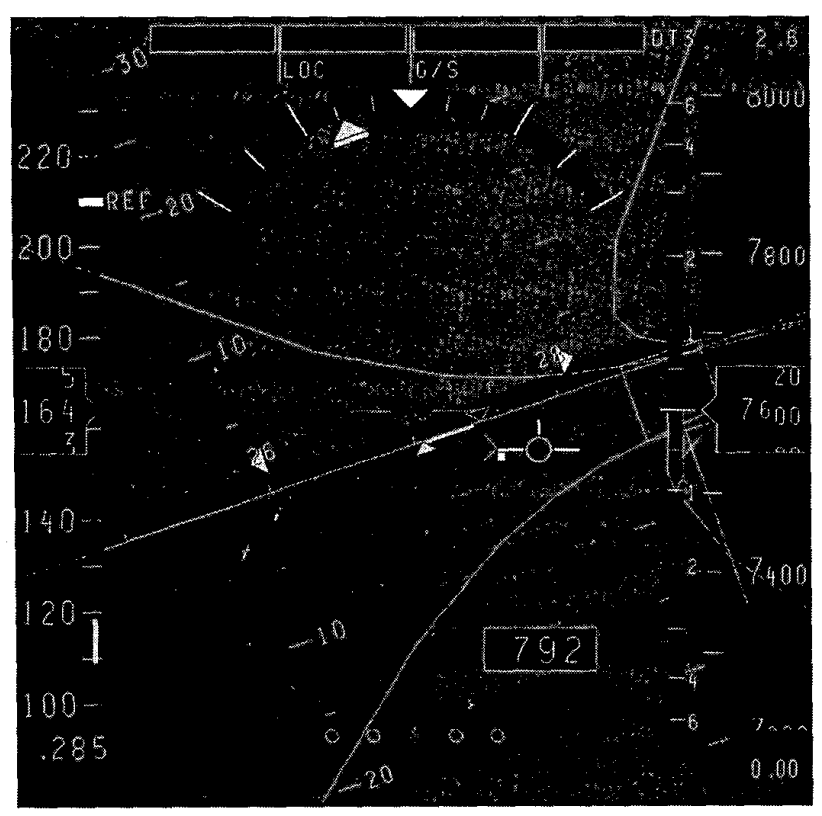

Fig. 1. Example of the PDF format with integrated terrain depiction

detect potential threats in the forward field of view; and during a manual landing, provides cues to the pilot for accurately flaring the aircraft. The out-of-the-windshield view does not provide a strategic overview, and unless the pilot is already familiar with the environment, he / she will also need maps and other navigation instruments. The SVS discussed in this paper also focuses on supporting the strategic information requirements.

\section{CONCEPT FOR THE SVIS}

To minimize the likelihood of CFIT, the SVS shall provide the pilot with continuous awareness of:

- Separation between the planned path and the terrain

- Deviation of the aircraft from the path

- Current and future expected separation between the aircraft and the terrain.

With good awareness in these areas, the pilot knows when the system is vulnerable, and in case of a problem does not have to build-up awareness to deal with the situation at hand.

To combine these safety benefits with increased operational capabilities, the SVS shall allow modes of operation that require complex trajectories in the presence of obstacles to be flown and need additional guidance as compared to current flight director (FD) displays and outside visual cues.

The information needed to perform a certain intended function is conveyed by one or more elements in a display format. When a single display shows multiple (separate) elements, each for a specific intended function, the format is sometimes referred to as integrated. Today's display formats focus on data extraction, in which the integration into information must be performed by the pilot. A goal of SVIS research is to actively support information integration, across tasks.

The following sections provide an overview of the functionality required for the VPD and the additional functionality required for the PFD and the ND relative to current state-of-the-art implementations.

\section{Primary Flight Display}

The PFD provides the information needed for precision manual guidance along a complex, conflict-free path. To support situation awareness, elements are included that provide predictability to the pilot as what to expect in terms of changes in the direction of the path and the distance between the path and terrain. By selecting the appropriate size of the pathway, the guidance concept allows the path to be flown to a specified lateral and vertical accuracy (quantified by the $95 \%$ containment volume for Flight Technical Error) with a high degree of repeatability and acceptable workload [3].

The depiction of the terrain is not intended for guidance, but designed to allow recognition of the locations where the separation between the planned path and the terrain can become an issue and to immediately see locations where a conflict with the terrain will occur when maintaining current direction of flight. To allow the pilot to assess the location of obstacles relative to the planned path, altitude droplines are depicted when the clearance between the path and the terrain is below a predefined threshold. To support the assessment of lateral distance toward obstacles and the ability to discriminate terrain features, a regular (checkerboard) texture pattern is used. Furthermore, like the ND, dynamic coloring of the terrain is used based on relative difference in altitude. Figure 1 shows an example of terrain in the PFD format.

The basic information needed for guidance is provided through the depiction of the future flight path as seen from the current position. The pilot derives the control actions from an understanding of guidance requirements, and, thus, has a basic level of navigation awareness. The preview on the future flight path allows the pilot to apply an anticipatory control strategy. The extent to which the pilot can benefit from this potential advantage depends on the accuracy with which the timing and magnitude of the required anticipatory control actions can be derived from the presentation. Since the image of the path results from the current aircraft position and attitude, whereas, on the control side, the pilot is confronted with higher-order system dynamics, the precision path tracking task will cause considerable workload. To compensate, some form of display augmentation is needed; four options will be discussed:

- Flight director

- Command aircraft

- Target flight path marker

- Position predictor. 


\section{Flight Director}

The flight director adds a command display that provides a compensatory tracking task. The dynamics can be optimized to compensate for latencies and higher-order system dynamics and provide good handling qualities. The pathway acts as a confirmatory source of information against which the commands provided by the FD can be compared.

\section{Command Aircraft}

A command aircraft serves as a position reference with which the pilot has to fly in formation. As such, it represents physically interpretable data. The commanded position provides outer-loop guidance commands. To compensate for higher-order system dynamics and latency, cues must be provided with which the pilot can close the inner-loop without generating a lot of lead. This can, for example, be accomplished by providing bank commands through orientation of the command aircraft. However, unlike the flight director and the predictor, the inner- and outer-loop cues are not integrated into a single dimension, thus, requiring mental integration by the pilot.

\section{Target Flight Path Marker}

Similar to the target flight path marker used in head-up displays, a target flight path marker can be used as a flight director to provide the pilot with a pursuit tracking task. The combination of this type of command data (which has no physically interpretable meaning, in the context of the status data provided by the perspective flightpath) may cause confusion. For example, to correctly fly a turn, the target flight path marker will always point a bit out of the turn.

\section{Position Predictor}

The use of a position predictor in a pathway display was first tested over twenty years ago [1]. The predictor and the path reference present the pilot with a pursuit tracking task. Like the FD control law, the prediction algorithm can be optimized to compensate for the effects of higher-order system dynamics. Furthermore, the position of the predictor, which is used for the control task, has a direct meaning, i.e., it represents physically interpretable information. It also supports the pilot in the application of an efficient anticipatory control strategy which reduces the control effort required for the compensatory tracking [3].

\section{Selecting an Augmentation Concept}

With an attitude-aligned perspective flight path display, the reference location of the flight path on the screen when flying the correct track varies as a function of the wind. When adding a two-bar FD to such a format, the reference condition for the FD is the center of the display. This can lead to a confusing presentation. Since the reference location of the predictor is related to the location of the flight path, this problem does not exist when using a flight path predictor. As indicated in the discussion about the command aircraft and the flight path target, both concepts have certain drawbacks. In contrast, the concept of the position predictor combines the advantage of the FD regarding the possibility to provide a single optimized guidance cue, with the advantage of the command aircraft because it provides information that can be directly related to the flight path itself. Given the excellent results, in terms of tracking performance, control activity $[1,3]$, and the possibility to apply anticipatory- and error-neglecting control strategies [3], the position predictor was selected.

\section{Representation of the Path}

As indicated earlier, the representation of the flight path must support the guidance task and support the pilot in maintaining awareness of the direction of the future path. The selection of values for the design parameters is discussed in [3]. To deal with clutter, the pathway is represented at multiple levels of detail, depending on the distance from the viewpoint.

\section{Special Functionality}

Special functionality has been developed to deal with degraded conditions during go-arounds, optimized tracking of turns, and interception of the planned path from an arbitrary point off the path.

In case of a go-around, the climb rate the aircraft can maintain depends on the energy state of the aircraft. To provide both lateral and vertical guidance during a missed approach procedure, the vertical position and slope of the path are based on the actual energy state of the aircraft. To allow optimized tracking of turns with tight position constraints, an algorithm can modify the curved path within certain constraints to allow the pilot to fly at a fixed bank angle in the presence of wind and still correctly roll out at the next straight segment. To provide support in situations where the pilot has to intercept the planned path, a trajectory, starting at the present position and providing guidance toward the desired intercept point, can be computed and displayed.

\section{Limitations}

It is recognized that, as a result of the limited field of view and the emphasis on near-term caused by the egocentric reference frame, the PFD is limited in its capability to provide the information necessary to assess the clearance between the path and the terrain beyond a near-term time horizon. The VPD and the ND are needed to provide this information.

\section{NAVIGATION DISPLAY}

The ND is a strategic display, and one of its functions is to support the pilot in the evaluation of proposed changes to the planned route. To keep the pilot aware of the location of potential obstacles in situations where he / she has to deviate from the planned path, because of ATC vectors, the ND includes color-coded terrain elevation information which allows the pilot to understand the contours of the terrain within the range selected on the ND. In case part of a planned path lies within a pre-defined limit from the terrain, this location is identified through color-coding of the path. Within a certain distance from 


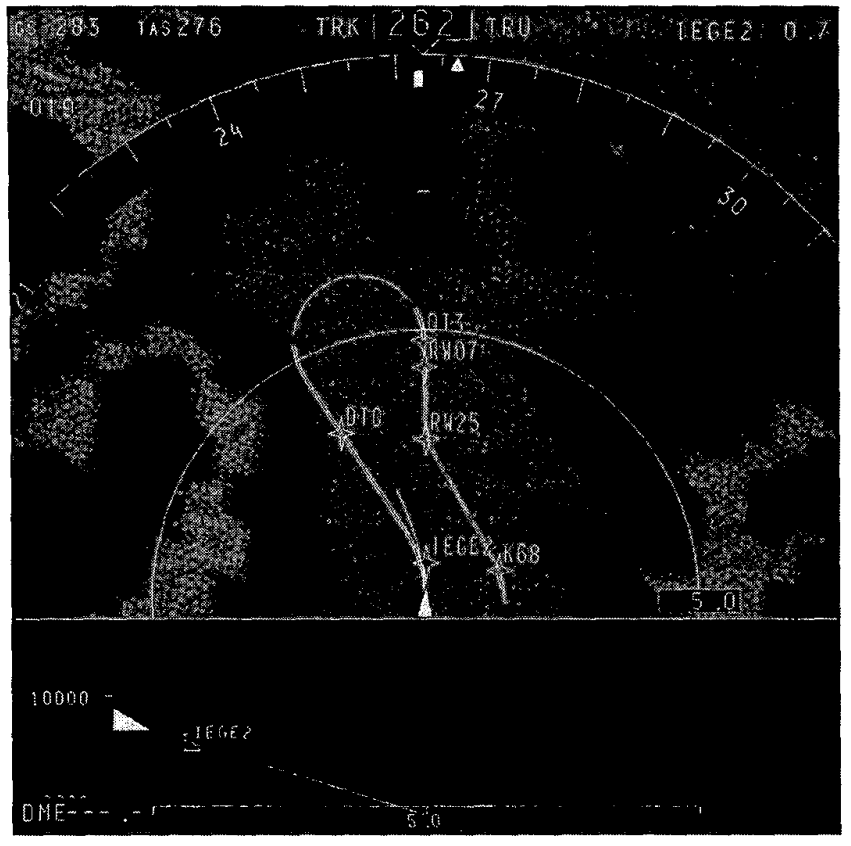

Fig. 2. ND with static sectional chart-based terrain elevation color-coding and dynamic color coding (green, yellow, and red) to convey information about relative height above the terrain

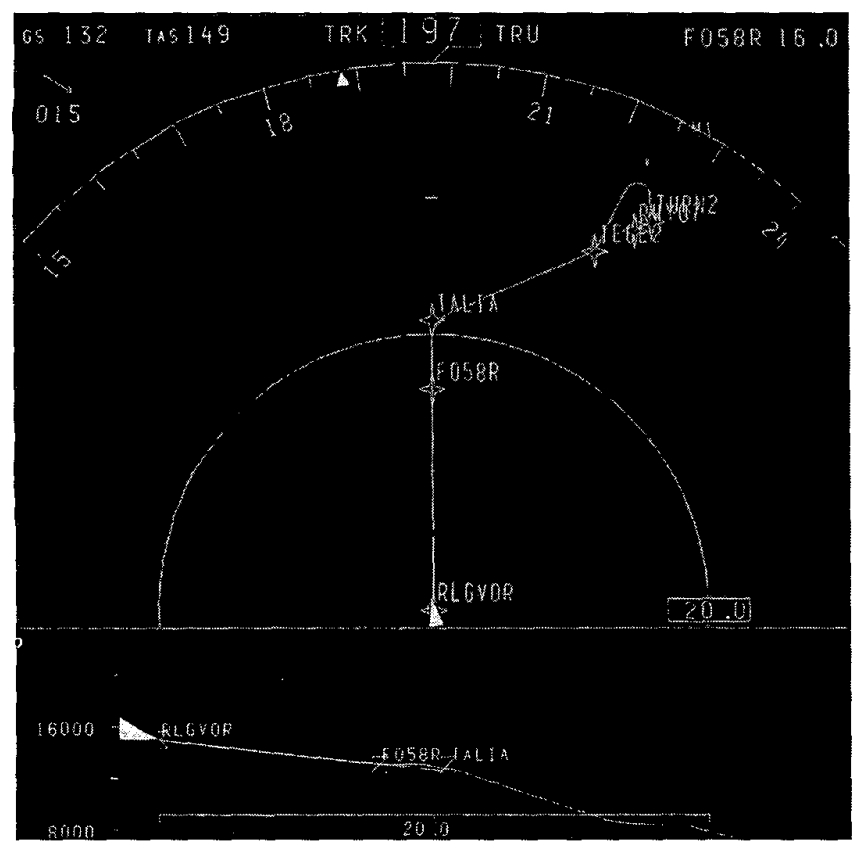

Fig. 3. ND with the VPD in the mode showing the terrain profile below the planned path

the terrain, the ND uses green, yellow, and red color-coding to indicate the altitude difference between the surrounding terrain and the altitude of the aircraft (see Figure 2).
To further support the pilot in case of a degraded aircraft condition, e.g. an engine failure, an energy-based prediction mode can be used. In this mode, dynamic terrain coloring is used to indicate the aircraft's ability to maintain a specified separation from the terrain. The computation is based on the energy state of the aircraft. Unlike the relative terrain coloring mode, in which more terrain will be colored when the aircraft descends, this mode immediately shows the areas that provide sufficient terrain clearance.

\section{VERTICAL PROFILE DISPLAY}

The Vertical Profile Display is intended to allow the pilot to instantaneously assess the clearance between the planned path and the terrain and the relation between the aircraft's vertical flight path and the planned path beyond the near-term preview provided by the PFD. It allows the pilot to timely detect conflicts between the planned path and the terrain in case of a managed mode or a hand-flown mode along a predefined path.

During a managed mode, with LNAV engaged, the VPD depicts the along-track path (Figure 3 ). In case of a deviation beyond predefined constraints, or in heading hold mode, the terrain in the direction of the current track is shown.

\section{EVALUATION}

The display formats have been evaluated by means of pilot-in-the-loop experiments, both in flight simulators and in laboratory aircraft. The simulator studies demonstrated an increase in navigational awareness and a reduction in task demand load of the perspective flight path display relative to a flight director display.

In 2000 and 2001, a series of flight trials was performed in the context of the NASA aviation safety program in the NASA 757 Airborne Research and Integrated Experiment System (ARIES). To show the experimental display formats to the evaluation pilots without having to remove or change the existing on-board displays, a research display was installed in the cockpit (see Figure 4).

During the 2001 flight trials performed at Eagle airport in Colorado, six pilots flew both NASA and Collins-team SVS display concepts. To rate performance and workload during this evaluation, both objective and subjective measures were used. The results of the flight tests confirmed that with the SVIS concept described in this paper, pilots can manually fly complex trajectories such as a continuous descending curved approach path (see Figure 5) with a small flight technical error.

The results from the workload assessment show that this is typically achieved with a very low mental, physical, and temporal demand on the pilot. Pilot comments also demonstrate that there was never any question of where they were, with respect to the path and the surrounding terrain. Regarding the use of the checkerboard texture on the terrain skin, several pilots commented that the terrain in the SVIS display did not seem as threatening as the same mountains did when seen from the cockpit. Whether, and if so, when this becomes an issue is currently being addressed. 


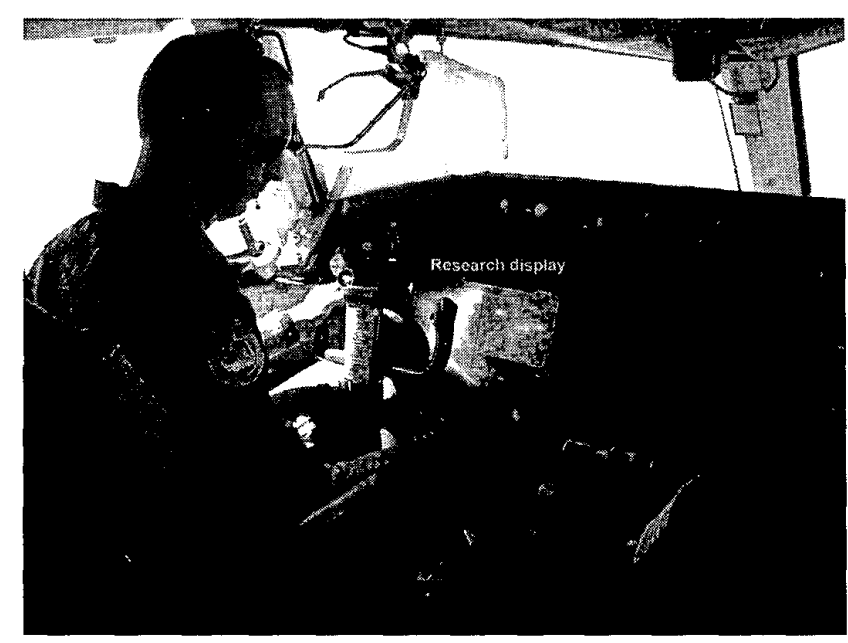

Fig. 4. Research display installed in the cockpit of the NASA 757 ARIES during an evaluation flight

\section{SUMMARY AND CONCLUSIONS}

The SVIS discussed in this paper comprises a number of elements that are additions to today's formats used on electronic flight displays. On the primary flight display, a perspective view on the future flight path and the terrain has been added, and the flight director has been replaced by a position predictor. On the lower part of the navigation display a vertical profile display has been added, showing a side view of the planned path relative to the terrain. On the navigation display, terrain elevation data has been added. Furthermore, functionality is integrated to support the pilot during missed approach procedures and navigation toward the planned path. The resulting SVIS has achieved the following goals:

- Provide continuous awareness of the surrounding terrain, both for strategic and tactical tasks.

- Increase navigation awareness by using a guidance concept in which the pilot derives control actions from an understanding of the future path and the position constraints.

- Reduce the effort necessary for information integration through integrated data presentation.

- Reduce task complexity and allow the pilot to anticipate changes in the guidance requirements by providing trajectory preview.

- Have a display augmentation concept compatible with the concept of using a depiction of the future flight path for guidance.

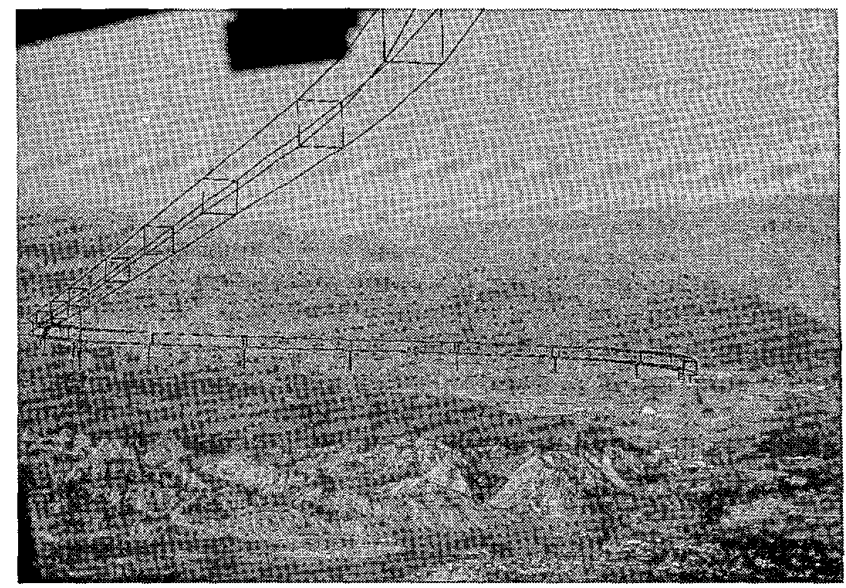

Fig. 5. Picture of the environment around Eagle Airport in Colorado. The tunnel that has been added to this picture shows the path of the continuous, curved descending approach to runway 07 that was flown with the SVIS display

\section{REFERENCES}

[1] Grunwald, A.J., J.B.Robertson and J.J.Hatfield, (1980). Evaluation of a Computer-Generated Perspective Tunnel Display for Flight-Path Following, NASA TP1736, Langley, VA

[2] Sachs, G. and H. Möller, (1995). Synthetic Vision Flight Tests for Precision Approach and Landing, Proceedings of the AIAA GNC Conference, August 7-10, Baltimore, MD.

[3] Theunissen, E., (1997). Integrated Design of a Man-Machine Interface for 4-D Navigation Delft University Press, ISBN 90-407-1406-1.

[4] Regal, D., (1991). Synthetic Vision in the Boeing High Speed Civil Transport, Proceedings of the 6th International Symposium on Aviation Psychology, pp. 198-203.

[5] Williams, D., M. Waller, J. Koelling, D. Burdette, T. Doyle, W. Capron, J. Barry and R. Gifford, (2001).

Concept of Operations for Commercial and Business Aircraft Synthetic Vision Systems Version 1.0.

[6] Baty, D.L., (1976). Rationale and Description of a Coordinated Cockpit Display for Aircraft Flight Management, NASA TM X-3457.

[7] Kuchar, J.J. and R.J.Hansman, (1993). Part-Task Simulator Evaluations of Advanced Terrain Displays, SAE Aerotech paper 932570 , Costa Mesa, CA

[8] Gordon, T.M., (2000), Synthetic Vision versus the Pilot's Existing View. Proceedings of the 19th DASC, pp. 2A1.1-2A1.8

[9] Theunissen, E., (1998),

Spatial Terrain Displays: Promises and Potential Pitfalls, Proceedings of the $17^{\text {th }}$ DASC. 